\title{
Large resistivity in numerical simulations of radially self-similar outflows
}

\section{Miljenko Čemeljić ${ }^{1}$, Nektarios Vlahakis ${ }^{2}$ and Kanaris Tsinganos ${ }^{2}$}

${ }^{1}$ Academia Sinica Institute of Astronomy and Astrophysics and Theoretical Institute for Advanced Research in Astrophysics, P.O. Box 23-141, Taipei, Taiwan email: miki@tiara.sinica.edu.tw

${ }^{2}$ IASA and Section of Astrophysics, Astronomy and Mechanics, Department of Physics, University of Atens, Panepistemiopolis 15784, Zografos, Athens, Greece email: vlahakis,tsingan@phys.uoa.gr

\begin{abstract}
We investigate conditions in a radially self-similar outflow in the regime of large resistivity. Using the PLUTO code, we performed simulations with proper choice of boundary conditions, relaxed at the footpoints of critical surfaces in the flow. We investigate outflow propagation in a high-resistive disk corona, and compare it to the results with small or vanishing resistivity.
\end{abstract}

Keywords. magnetic fields (magnetohydrodynamics): MHD, methods: numerical, stars: winds, outflows.

\section{Initial and boundary conditions}

General classes of self-consistent ideal-MHD solutions have been constructed in Vlahakis \& Tsinganos (1998). In Gracia et al. 2006 ideal-MHD solutions with modified
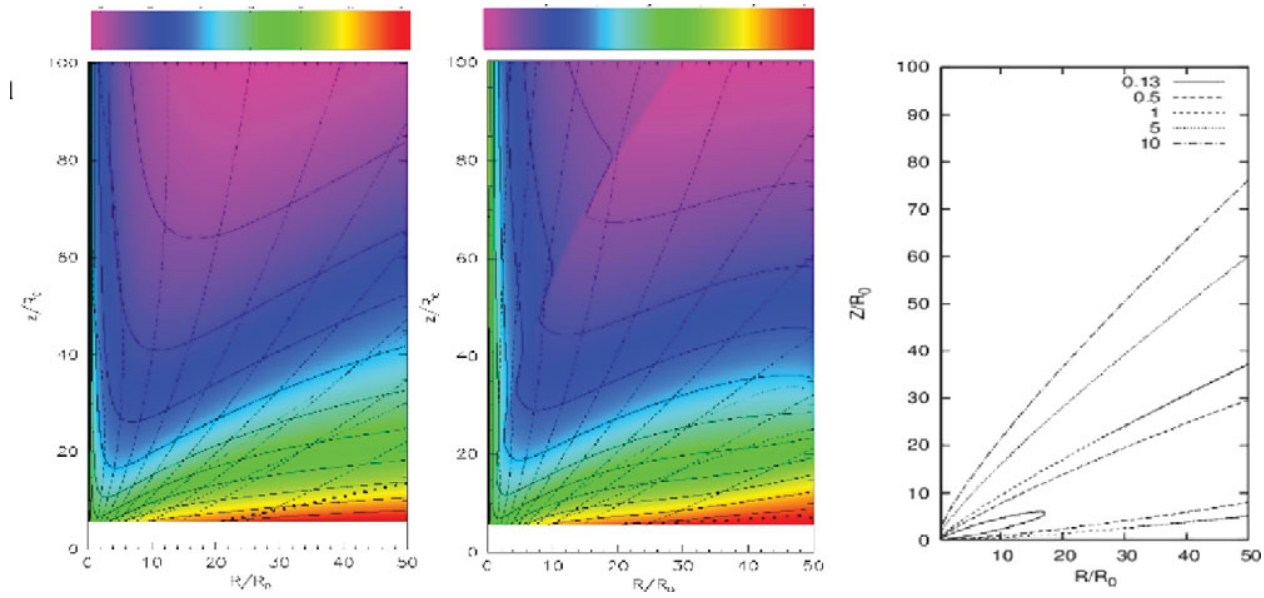

Figure 1. Initial state in our NIRVANA simulations for ideal-MHD and for simulations with not too large resistivity is shown in the leftmost panel. Logarithmic color grading and solid lines represent density, magnetic field lines are shown in dashed lines, and critical surfaces are shown in dotted lines. The density, magnetic field and velocity are slightly modified from the analytical solutions for numerical reasons. In the middle panel is shown the final, stationary state in low resistivity simulations, which does not differ significantly from the initial state. In the rightmost panel is shown the value of $0.5 \beta\left(V R / V_{0} R_{0}\right)$ for the analytical solution, which gives critical value of resistivity that corresponds to $\mathrm{Rb}$. We introduced a new number, describing influence of the magnetic diffusivity $\eta$ on the energy transport, which can be written in terms of Rm and plasma beta as $\mathrm{Rb}=\mathrm{Rm} \beta / 2$. 

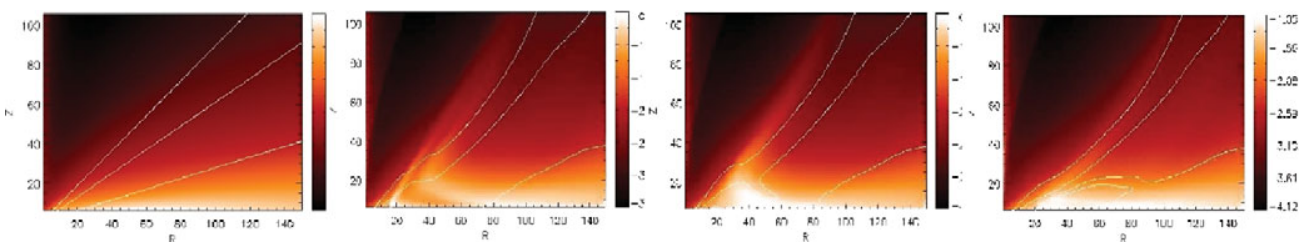

Figure 2. To avoid influence of the outer boundary, we triple the computational domain in $\mathrm{R}$ direction, and results are taken before the reflected wave can return from the outer R-boundary to our domain of interest, $\mathrm{RxZ}=50 \times 100$. We show results for large resistivity, one order of magnitude above the critical resistivity found in previous study. Boundary conditions are not over-specified and solutions are different for different b.c. relaxed, what could infer on the outcome of simulations, and we study it closer. Here we show the initial density and critical surfaces, and results after the relaxation for $\mathrm{Vx}, \mathrm{Vy}, \mathrm{Bz}$ boundary conditions relaxed, left to right respectively, for $\eta=1.5$.

analytical initial conditions were obtained, and followed with extension in the resistiveMHD in Cemeljic et al. 2008-both using the NIRVANA code (version 2.0, Ziegler (1998)).

Solutions with small and moderate resistivity show smooth departure from the idealMHD solutions as shown in Fig. 1, up to critical resistivity, for which solutions changed abruptly — see Fig. 2. This critical resistivity was $10^{3}$ times larger than numerical resistivity in simulations. Here we investigate the case with large resistivity, solving the resistive-MHD equations with the diffusive terms added in the induction and energy equations, by the PLUTO code by Mignone et al. 2007. Anomalous resistivity often introduced in simulations is usually few orders of magnitude larger than microscopic resistivity, and it is instructive to investigate how a code treats such problem.

\section{Results}

We show, for the first time, results of resistive simulations above the critical resistivity found in our previous study, in comparison with the analytical solution of the closely related ideal-MHD problem. Understanding those results, and disentangling the physical and numerical causes for differences, is essential for understanding the simulations of launching and propagation of resistive astrophysical jets.

\section{References}

Čemeljić, M., Gracia, J., Vlahakis, N., \& Tsinganos, K. 2008, MNRAS, 389, 1022

Gracia, J., Vlahakis, N., \& Tsinganos, K. 2006, MNRAS, 367, 201

Mignone, A., Bodo, G., Massaglia, S., Matsakos, T., Tesileanu, O., Zanni, C., \& Ferrari, A. 2007, APESS $, 287,129$

Vlahakis, N. \& Tsinganos, K. 1998, MNRAS, 298, 777

Ziegler, U. 1998, Co.Ph.Com, 109, 111 